\title{
IoT Based Smart Farming in a Agriculture
}

\author{
Vijayalakshmi M. M \\ Assistant Professor, \\ Department of Information Science and Engineering, \\ GSSSIETW, Mysuru, Karnataka, India
}

\begin{abstract}
Internet of Things (IOT) advances can be used in smart farming to enhance quality of agriculture. Agriculture the backbone of Indian economy, contributes to the overall economic growth of the country. The paper aims making use of evolving technology. Monitoring environmental factors is the major factor to improve the yield of the efficient crops. The features of this paper includes detection of leaf disease, server based remote monitoring system, temperature sensing, Soil Moisture Sensing etc. It makes use of sensors networks for measurement of moisture, temperature and humidity instead of manual check. Various Sensors are deployed in various locations of farms, to control all these sensors it has been used one controller called Raspberry PI (RPI) and pic microcontroller. Leaf disease can be detected by the camera interfacing with RPI. Immediate status of a farm like a leaf disease and other environmental factors affecting crop like temperature and moisture is sent using WIFI Server through RPI to the farmers and also message alert will be sent to the owner about the field conditions through mobile.
\end{abstract}

\section{KEYWORDS: Ultrasonic Sensor, Pi Camera, Temperature and Moisture Sensors, Raspberry Pi and Pic Micro Controller}

\section{INTRODUCTION}

Internet of Things (IoT) is widely used in connecting devices and collecting data information. Internet of Things is used with IoT frameworks to handle and interact with data and information. In the system users can register their sensors, create streams of data and process information. IoT are applicable in various methodologies of agriculture. Applications of IoT are Smart Cities, Smart Environment, Smart Water,Smart Metering, Security and Emergency, Industrial Control, Smart Agriculture, Home Automation, eHealth etc. 'Internet of Things' is based on device which is capable of analysing the sensed information and then transmitting it to the user.

Why agriculture needs IOT?

From survey of United Nations - Food and Agriculture Organisations, the world wide food production should be increased by $70 \%$ in 2050 for evolving population. Agriculture is the basis for the human species as it is the main source of food and it plays important role in the growth of country's economy.

It also gives large ample employment opportunities to the people. The farmers are still using traditional methods for agriculture which results in less yielding of crops and fruits.So the crop yield can be improved by using automatic machineries. There is need to implement modern science and technology in the agriculture for increasing the yield. By using IoT increase in production with low cost by monitoring the efficiency of the soil, temperature and humidity monitoring, rain fall monitoring, fertilizers efficiency, monitoring storage capacity of water tanks and also theft detection in agriculture areas.

The combination of several technologies as Internet of Things and wireless sensors networks can lead to agriculture modernization. The wireless sensor network which collects the data from different type of sensors and send it to the main server wireless protocol.
There are many other factors that affect the productivity to great extent. Factors include attack of insects and pests which can be controlled by spraying the proper insecticide and pesticides and also attack of wild animals and birds when the crop grows up. The crop yield is declining because of unpredictable monsoon rainfalls, water scarcity and improper water usage.

The collected data provide the information about the various environmental factors. Monitoring the environmental factors is not the complete solution to increase the yield of crops. There are number of other factors that decrease the productivity to a greater extent. Hence automation must be implemented in agriculture to overcome these problems. So, in order to provide solution to all such problems, it is necessary to develop an integrated system which will take care of all factors affecting the productivity in every stage. But complete automation in agriculture is not achieved due to various issues.Though it is implemented in the research Level it is not given to the farmers as a product to get benifitted from the resources.Hence it deals about developing smart agriculture using IOT and given to the farmers.

\section{RELATED WORKS}

The recent trends in implementation of IOT in the smart farming techniques. In the meantime, a brief literary survey has been made on the published works of eminent scholars in this field.

In [1], smart precision based agriculture makes use of wireless sensor networks to monitor the agricultural environment.Zigbee and raspberry pi-based agriculture monitoring system serves as a reliable and efficient method for monitoring agricultural parameters. Wireless monitoring of field not only allows user to reduce the human power, but it also allows user to see accurate changes in it. It focuses on developing devices and 
tools to manage, display and alert the users using the advantages of a wireless sensor network system. A smart system based on precision agriculture would pave the way to a new revolution in agriculture.

In [2], a smart way of automating farming process can be called as Smart Agriculture. By implying an automated system threats can be eliminate possible threats to the crops by reducing the human intervention. Environment real time monitoring is an important factor in smart farming. GUI based software will be provided to control the hardware system and the system will be entirely isolated environment, equipped with sensors like temperature sensor, humidity sensor, photo emitter.This system will realize smart solution for agriculture and efficiently solve the issues related to farmers.The environment will not be the barrier for production and growth of any plant and can overcome the problem of scarcity of farming production.

In [3], a low cost and efficient wireless sensor network technique to acquire the soil moisture and temperature from various locations of farm and as per the need of crop controller take the decision to make irrigation $\mathrm{ON}$ or $\mathrm{OFF}$

In [4], the system was powered by photovoltaic panels and had a duplex communication link based on a cellularInternet interface that allowed for data inspection and irrigation scheduling to be programmed through a web page.

In [5], zigbee protocols based on IEEE 802.15.4-2003 for wireless system are used. The atmospheric conditions are monitored and controlled online by using Ethernet IEEE 802.3.Partial Root Zone Drying Process can be implemented to save water at the maximum extent. Online interaction can be made with the farmers by the consultant to give them the knowledge about this technique and implement it effectively in their farms to extract more yield with advanced technology.The technological development in Wireless Sensor Networks made it possible to use in monitoring and control of greenhouse parameter in precision agriculture.

In [6], climate change and rainfall has been erratic over the past few decades. Due to this in recent era, climate smart methods called as smart agriculture is adopted by many Indian farmers. Smart agriculture is an automated and directed information technology implemented with IOT (Internet of Things). IOT is developing rapidly and widely applied in all wireless environments. The major objective is to get the real-time data and reduce the water that is lost in the irrigation process and reduce the time spent on the field.

In [7][8][9], Proposed smart irrigation systems using Internet of Things. To calculate humidity and water levels of soil some wireless sensors are needed. These sensed data are sent to a smart gateway through a network, using agatewaycalled Generic IoT Border Router Wireless $\mathrm{Br}$ 1000. From the gateway, the data is then sending to a web service through a network.Conducted a survey on Smart Agriculture Irrigation systems to get better understand about the IoT-based development in agriculture with cloud computing.
In [10], proposed a greenhouse Monitoring System based on agriculture IoT with a cloud. In a greenhouse, management can monitor different environmental parameters effectively using sensor devices such as light sensor, temperature sensor, relative humidity sensor and soil moisture sensor. Periodically ( 30 seconds) the sensors are collecting information of agriculture field area and are being logged and stored online using cloud computing and Internet of Things.

In [11], paper explain an IOT Based Crop-Field Monitoring and Irrigation Automation system. In their work, to monitor crop-field a system is developed by using sensors and according to the decision from a server based on sensed data, the irrigation system automated. By using wireless transmission the sensed data forwarded towards to web server database. If irrigation is automated then that means if the moisture and temperature fields fall below of the potential range. The user can monitor and control the system remotely with the help of application which provides a web interface to the user.

In [12] [13] [14], The detection of plant leaf is an very important factor to prevent serious outbreak.Automatic detection of plant disease is essential research topic. In digital image processing, various algorithms are utilized to perform the different image processing steps on digital images. In agricultural area, digital image processing methods are mostly used for the purpose of plant growth monitoring, plant disease prediction, and so on.A method for detection and classification of leaf diseases is implemented. The segmentation of the diseased part is done using K-Means segmentation. In plants, diseases can be found in various parts such as fruits, stems and leaves. The main diseases of plants are viral, fungus and bacterial disease like Alternaria, Anthracnose, bacterial spot, canker, etc.,.The viral disease is due to environmental changes, fungus disease is due to the presence of fungus in the leaf and bacterial disease is due to presence of germs in leaf or plants. The proposed framework can be used to identify leaf diseases.Automatic detection of plant diseases is an important research topic since it is able to automatically detect the diseases from the symptoms that appear on the plant leaves.

\section{PROPOSED PROBLEM STATEMENT}

This paper present proposed model for smart agriculture to develop real time monitoring system for soil properties like temperature, moisture, water level and crop disease identification using image analysis and SMS based alerts. It will also be possible to control various operation of the filed remotely from anywhere, anytime by mobile as well as web application.

\section{PROPOSED ARCHITECTURE}

So to overcome this problem smart agriculture techniques using IOT as been introduced.This paper includes various features like moisture \& temperature sensing, intruders detecting, security, leaf dieases detection and proper irrigation facilities. It makes use of wireless sensor networks for noting the soil properties and environmental factors continuously. Various sensor nodes are deployed at different locations in the farm. Controlling these parameters are through any remote device or internetservices and the operations are performed by interfacing sensors, camera with microcontroller. 
Proposed system has three modules Intruder detection module, Leaf diseases detection module and temperature and sensor module.

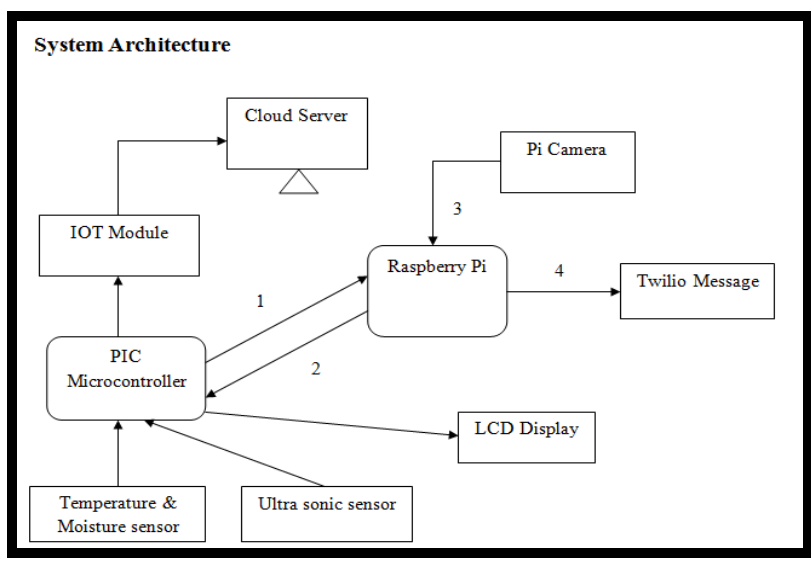

Fig.1.Smart Farming System Architecture Diagram

In Fig-1, Initially the sensors like temparatue, moisture sensors and ultrasonic sensor is connected to the pic microcontroller where it places in a field of agriculture. By using pic microcontroller can control all these sensors is connected to Raspberry pi.The temperature sensor will sense the temperature around the atmosphere and send it to the microcontroller.

The moisture sensor senses the moisture content in the soil and sends moisture level of the soil to the microcontroller.

The ultrasonic sensor detects the intruder or any unknown persons are entering into the field, if any intruder detected by the sensor then the buzzer will on and generate an alert to the surrounding people.

In the field section various sensors are deployed in the field like temperature sensors, moisture sensor and ultrasonic sensor. The data collected from these sensors are connected to the microcontroller through RS232.

In control section, the received data is verified with the threshold values. If the data exceeds the threshold value the buzzer is switched ON and the LED starts to blink. This alarm is sent as a message to the farmer and automatically the power is switched OFF after sensing. This collected data from the pic microcontroller is sent to the cloud using ESP8266 (IOT module).

The data will be regularly updated in the Things speak website connected through the IOT module. In automatic mode, the microcontroller gets switched $\mathrm{ON}$ and OFF automatically if the value exceeds the threshold point.Soon after the microcontroller is started, automatically an alert must be sent to the user through twilio account to the mobile. This is achieved by sending data to raspberry pi. Other parameters like leaf disease detection is done using pi camera present in the raspberry pi.

\section{A. Intruder Detection Module}

The functionality is used to detect the intruder in the field. This can be implemented using ultrasonic sensors. Ultrasonic sensors detect the distance of intruder and sends the messages to mobile. This helps in intruder detecting in the farm through the distance and also the buzzer will be on.

\section{B. Leaf Disease Detection Module}

This module consists of Pi camera. It captures the image of the leaf and these images are compared with the prestored images of database. First, the real time images of various leaves are acquired using a pi camera. Camera interfacing with raspberry pi is very easy.

The Raspberry pi board mostly is used to acquired the images of the crops from camera output. Raspberry pi perfect for any automation. Then various image-processing methods are applied to the acquired images to getting useful features that are important from next analysis process. Preprocessing is used to convert from RGB to grey, as grey scale image gives perfect accuracy to defect detection.

Feature extraction process will consists actual disease detection from an image by comparing the image with nondefected images. After that, many analytical techniques or methods are carried out to classify the images according to the particular problem at hand. Finally detected and classified disease will be send to the mobile phone. The image of leaf is capture by camera acquired leaf images are converted into gray image.

\section{Temperature And Moisture Sensor Module}

This function will consist of Temperature sensor and moisture sensor.When the temperature exceeds the threshold range and also the moisture content in the soil below the threshold level then the pumping motor will on automatically and it send an alert message to the owner. Temperature Sensors measure the amount of heat energy or even coldness that is generated by an object or system, allowing us to sense or detect any physical change to that temperature producing either an analogue or digital output. There are many different types of Temperature Sensor available and all have different characteristics depending upon their actual application.Soil moisture sensors measure the volumetric water content in the soil.

\section{RESULTS}

In this section some of the screenshots of the project has been displayed.

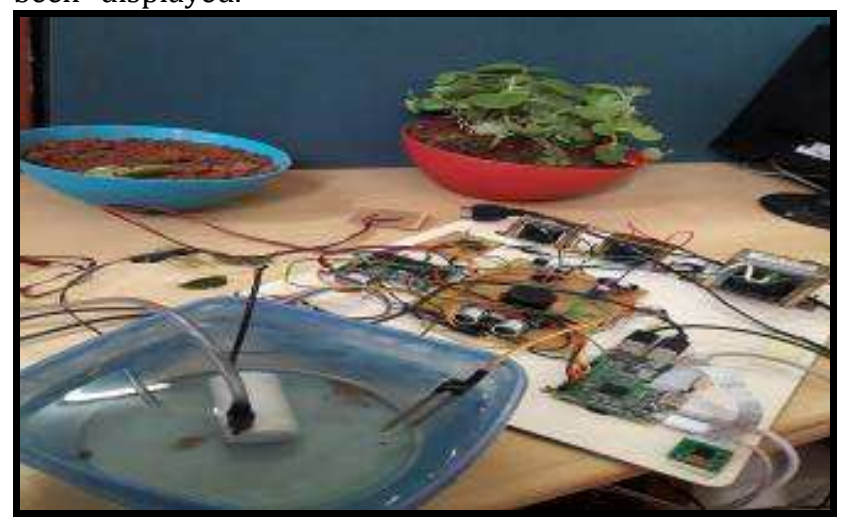

Fig-2: Overall project model

The above fig- 2 is the overall look of theproject consists of raspberry pi, pic microcontroller, sensors which is used for smart farming in the agriculture field.These sensors are connected to picmicrocontroller which is responsible for 0 controlling of these sensors.Threshold values are fixed based on the sensors.For example if the threshold value of 
the moisture sensor exceeds then water will be pumped to the field to maintain moisture level.

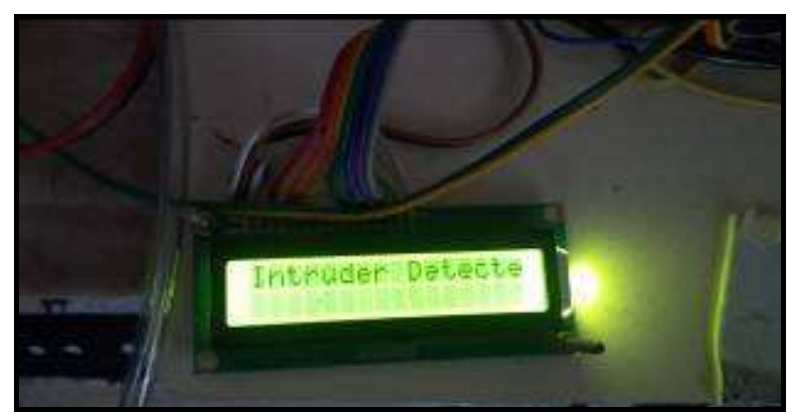

Fig-3: Intruder Detection

The above Fig-3, indicates the intruder detection using the ultrasonic sensors. In the field if any intruder is detected automatic buzzer will be $\mathrm{ON}$ and same will be displayed on the LCD screen.

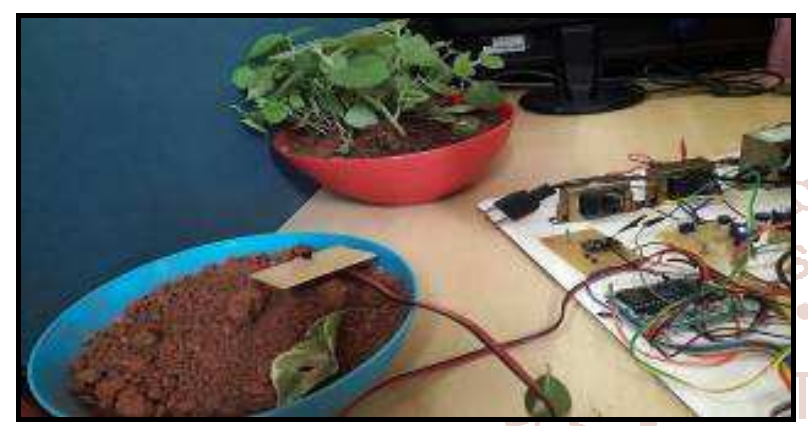

Fig-4: Temperature level Detection

The above Fig-4, shows temperature sensor LM335.the temperature sensors will be kept in the field to sense the sourrounding temperature. Based on temperature value the protection of crops can take care.

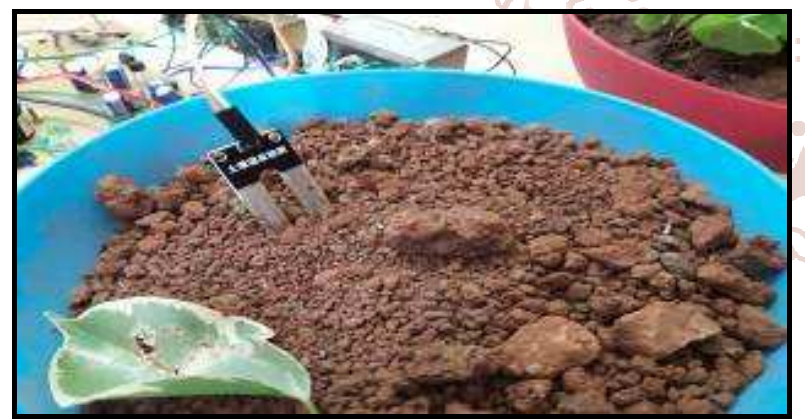

Fig-5: Moisture Detection

The above Fig-5, shows moisure sensor in the dry soil. Moisture sensor sense the moisture content of the soil and based on the value automatic water pump will be $\mathrm{ON}$ to increase the moisture content.

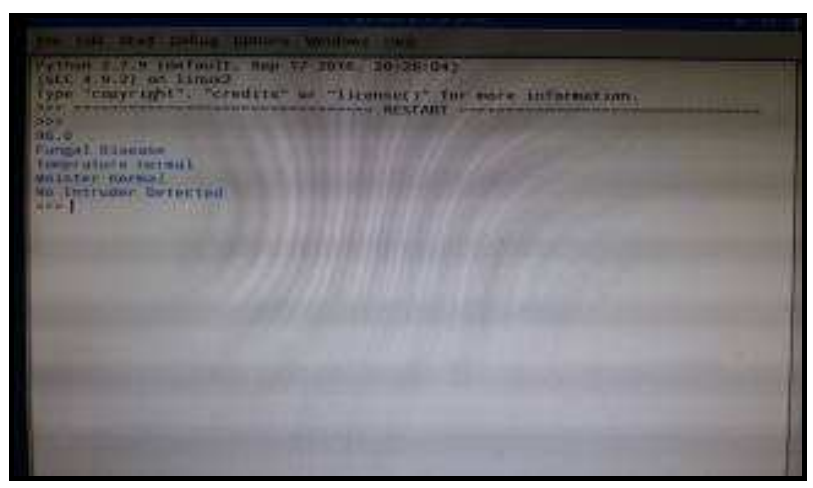

Fig-6: Leaf Disease Detection
The above Fig- 6 indicates the disease of the leaf for a given plant using pi camera attached to raspberry pi. Based on the image processing technique particular disease of the leaf will detected. This page also displays the temperature and moisture and intruder detection in the given field.

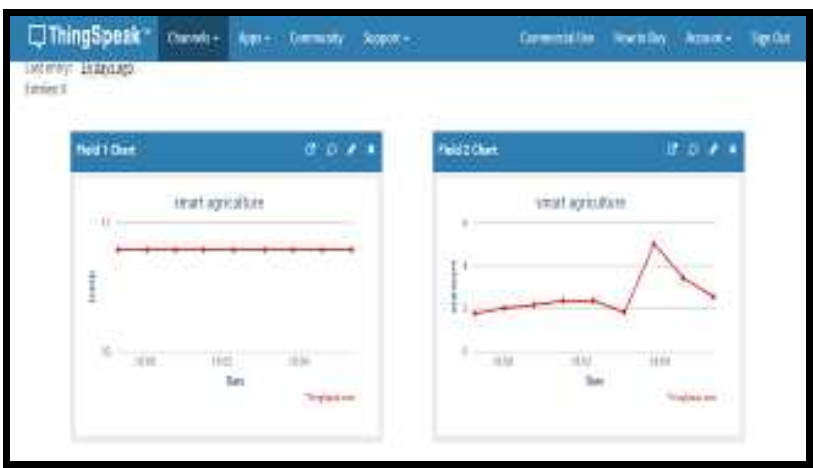

Fig-7: Thingspeak Account

The above fig-7 shows daily report of the field based on the moisture, temperature and intruder detection.The graph indicates the variations of temperature and moisture in the field.Things speak is the website used to retrieve the field data.

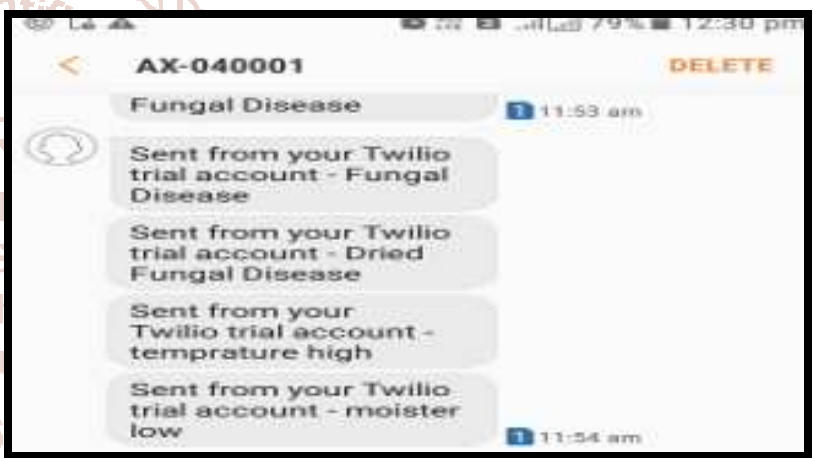

Fig-8: Messages From Twilio Account To Mobile

The above fig- 8 shows the screenshot of the mobile screen. This indicates the alert messages obtain from the field based on the sensor values send to the farmer through twilio account.

\section{CONCLUSION}

The use of automated monitoring and management system are gaining increasing demand with the technological advancement. In agricultural field loss of yield mainly occurs due to widespread of disease and traditional farming techniques.Mostly the detection and identification of the disease is noticed when the disease advances to severe stage. The proposed system is capable of detecting the field conditions like soil moisture content, temperature condition,intruder detection and the leaf disease at the earlier stage as soon as it occurs on the leaf. Hence saving the loss and reducing the dependency on the expert to a certain extent is possible. It can provide the help for a person having less knowledge about the disease. Depending on these goals extract the features corresponding to the disease.

\section{FUTURE WORK}

Large potential of our Indian agriculture is yet untapped but still it has miles to travel in this arena of research as different types of soil textures in different regions of our state. Farmers can be benefitted by the actual 
implementation of this projected program. Real challenges that were faced and that are yet to be overcome in reality are the internetworking of the nodes in an agricultural field and in designing a user friendly application that is easily understandable language for farmers.

\section{REFERENCES}

[1] K. Lakshmisudha, swathi Hegde, Neha Kale, Shruti Iyer, "Smart Precision Based Agriculture Using0Sensors", International0Journal of Computer Applications (09758887), Volume 146-No.11, July 2011

[2] Nikesh0Gondchawar, Dr. R. S. Kawitkar, "IoT Based Smart Agriculture", International Journal of Advanced Research in Computer and Communication Engineering (IJARCCE), Vol.5, Issue 6, June 2016.

[3] M. K. Gayatri, J. Jayasakthi, Dr. G. S. Anandhamala, "Providing Smart Agriculture Solutions to Farmers for Better Yielding Using IoT", IEEE International Conference on Technological Innovations in ICT for Agriculture and Rural Development (TIAR 2015).

[4] Chetan Dwarkani M, Ganesh0Ram R, Jagannathan S, R. Priyatharshini, "Smart Farming System Using Sensors for Agricultural Task Automation", IEEE International Conference on Technological Innovations in ICT for Agriculture and Rural Development (TIAR 2015).

[5] S. R. Nandurkar, V. R. Thool, R. C. Thool, "Design and Development of Precision Agriculture System Using Wireless Sensor Network", IEEE International Conference on Automation, Control, Energy and Systems (ACES), 2014.

[6] Joaquín Gutiérrez, Juan Francisco Villa-Medina, Alejandra Nieto-Garibay, and Miguel Ángel PortaGándara, "Automated Irrigation System Using a Wireless Sensor Network and GPRS Module", IEEE
Transactions on Instrumentation and Measurements, 0018-9456,2013.

[7] Prathibha S R, Anupama Hongal and Jyothi M P," Iot Based Monitoring System In Smart Agriculture" 2017 International Conference on Recent Advances in Electronics and Communication Technology.

[8] Mahesh M. Galgalikar "Real-Time Automization of Agricultural Environment for Social Modernization of Indian Agricultural System" 2010 IEEE

[9] K. A. Patil and N. R Kale "A Model for Smart Agriculture Using IoT" 2016 International Conference on Global Trends in Signal Processing, Information Computing and Communication

[10] Mahammad Shareef Mekala and Dr P. Viswanathan "A Survey : Smart Agriculture IoT with Cloud Computing”, 2017 IEEE

[11] Gautam Kaushal and Rajni Bala "GLCM and KNN based Algorithm for Plant Disease Detection" International Journal of Advanced Research in Electrical, Electronics and Instrumentation Engineering Vol. 6, Issue 7, July 2017

[12] M. Reyalat, Al Hiary, Bani Ahmad, Braik and Z. Rahamneh, "Fast and Accurate Detection0and Classification of Plant Diseases".

[13] Sanjay B. Dhaygude, Nitin P. Kumbharv "Agricultural plant Leaf Disease Detection Using Image Processing ", International Journal of Advanced Research in Electrical, Electronics and Instrumentation Engineering Vol. 2, Issue 1, January 2013

[14] R. Shripriya1, N. Yuvaraj," A survey on leaf disease prediction algorithms using digital image processing", Indian Journal of Innovations and Developments Vol5(10), October, 2006. 